\title{
The role of vitamins in the diet of the elderly II. Water-soluble vitamins
}

\author{
J. Csapó ${ }^{1,2}$ \\ e-mail: csapo.janos@gmail.hu \\ Cs. Albert ${ }^{1}$ \\ e-mail: albertcsilla@cs.sapientia.ro
}

\author{
J. Prokisch ${ }^{2}$ \\ e-mail: jprokisch@agr.unideb.hu
}

${ }^{1}$ Sapientia Hungarian University of Transylvania, Faculty of Miercurea Ciuc, Department of Food Science, RO-4100 Miercurea Ciuc, Piata Libertăţii 1., Romania ${ }^{2}$ University of Debrecen, Faculty of Agricultural and Food Sciences and Environmental Management, Institute of Food Technology, H-4032 Debrecen, Böszörményi St 138., Hungary

\begin{abstract}
Following a presentation of humans' water-soluble vitamin requirements, the authors will discuss in detail the role these vitamins play in human organism and outline those major biochemical processes that are negatively affected in the body in case of vitamin deficiency. They point out that in the elderly population of developed countries cases of water-soluble vitamin deficiency are extremely rare and they are due to the lack of dietary vitamin, but mostly to the vitamin being released from its bindings, the difficulty of free vitamin absorption, gastrointestinal problems, medication, and often alcoholism. Among water-soluble vitamins, $\mathrm{B}_{12}$ is the only one with a sufficient storage level in the body, capable of preventing deficiency symptoms for a long period of time in
\end{abstract}

Keywords and phrases: $B$ vitamins, vitamin $B_{1}, B_{2}, B_{3}, B_{6}, B_{12}$, thiamine, riboflavin, niacin, folic acid, biotin, pantothenic acid, vitamin $\mathrm{C}$, ascorbic acid, vitamin $\mathrm{U}$, water-soluble vitamins, vitamin requirements, vitamin requirements of the elderly 
cases of vitamin-deficient nutrition. Each type of vitamin is dealt with separately in discussing the beneficial outcomes of their overconsumption regarding health, while the authors of the article also present cases with contradictory results. Daily requirements are set forth for every watersoluble vitamin and information is provided on the types of nutrients that help us to the water-soluble vitamins essential for the organism.

\section{Introduction}

Our previous review article reports on humans' protein and energy needs, the role of fat-soluble vitamins in the diet of elderly people. The present paper will treat the water-soluble vitamin needs of elderly people. Beyond generalities, we will discuss the role of each vitamin, the consequences of vitamin deficiency and overuse, and the therapeutic use of vitamins, in each case touching upon the needs of elderly people. Table 1 shows the RDI values of water-soluble vitamins and the tolerable upper limits (Thomas, 2007).

Table 1: RDI values of water-soluble vitamins for the male and female population over 70 years of age and the tolerable upper limits

\begin{tabular}{|c|c|c|c|}
\hline Vitamin & Men & Women & Upper limit \\
\hline Vitamin $\mathrm{B}_{1}$ (Thiamine) $(\mathrm{mg})$ & 1.2 & 1.1 & no data \\
\hline Vitamin $B_{2}$ (Riboflavin) (mg) & 1.3 & 1.1 & no data \\
\hline Vitamin $B_{3}$ (Niacin) (mg) & 16 & 14 & 35 \\
\hline Vitamin $\mathrm{B}_{6}$ (Pyridoxine) (mg) & 1.7 & 1.5 & 100 \\
\hline Vitamin $\mathrm{B}_{12}$ (Cyanocobalamin) $(\mu \mathrm{g})$ & 2.4 & 2.4 & no data \\
\hline Folic acid (mg) & 600 & 600 & 1,000 \\
\hline Biotin (mg) & 30 & 30 & no data \\
\hline Pantothenic acid (mg) & 5 & 5 & no data \\
\hline Vitamin C (Ascorbic acid) (mg) & 90 & 75 & 2,000 \\
\hline
\end{tabular}

If the data included in the above table were compared with levels determined for younger people, we could only observe minimal differences, which reminds us of the fact that only certain types of disease as well as cases of indigestion and malabsorption need special attention regarding the vitamin needs of the elderly population. 


\section{Vitamin requirements}

Our body does not store up water-soluble vitamins, wherefore it falls to us to take care of the daily vitamin B supply - except for vitamin $\mathrm{B}_{12}$ stored in the liver. Due to the facts outlined above, deficiency for vitamin $B_{1}$ was found in $13-43 \%$ and for vitamin $\mathrm{B}_{6}$ in 5-56\% of the elderly people (Hajjar \& Pharm, 2007). Such typical cases of vitamin deficiency diseases as scurvy, beriberi, or pellagra have a very low incidence rate among the senior population. Vitamin deficiencies tend to be symptomatic in cases of insufficient consumption of foods in terms of quantity and quality or when people refuse to consume certain types of food on a whim (Gordon, 1993).

\section{Water-soluble vitamins}

\subsection{Vitamin $\mathrm{B}_{1}$}

The first member of the B-group vitamins, vitamin $\mathrm{B}_{1}$ (thiamine, aneurine, thiamine pyrophosphate, TPP), is the coenzyme of enzymes that take part in the citric acid cycle and help the release of chemical energy. The decarboxylation of the pyruvic acid and $\alpha$-ketoglutaric acid into acetyl-coenzyme $\mathrm{A}$ and succinyl-coenzyme A are key reactions of this cycle, while both enzymes function with TPP coenzyme. Key enzymes containing TPP can also be found in the pentose phosphate cycle as well as in the synthesis of ATP, deoxyribonucleic acid (DNA), and ribonucleic acid (RNA), listing it as an indispensable vitamin for our organism. Ascertainment of our body's vitamin $B_{1}$ supply can take place via the measurement of erythrocyte transketolase activity or, more recently, via the direct analysis of erythrocyte TPP content.

Vitamin $\mathrm{B}_{1}$ deficiency causes beriberi disease. Typical symptoms of this avitaminosis are: neuritis; muscle weakness; insomnia; oedema starting in the extremities, and ultimately covering the entire human body; finally, death would set in due to paralyses and cardiac dysfunction. As a consequence of vitamin $\mathrm{B}_{1}$ deficiency, carbohydrate metabolism is disrupted as the intermediary metabolites (pyruvic acid, lactic acid) become concentrated in the tissues and the blood, since the decomposition of the pyruvic acid takes place with the aid of an enzyme containing vitamin $B_{1}$. Linked to phosphates, vitamin $\mathrm{B}_{1}$ forms thiamine pyrophosphate (TPP) coenzyme - in the case of its deficiency, a dysfunction in the pyruvic acid decarboxylase and transketolase sets in, whose prosthetic group. 
Based on its structure, vitamin $\mathrm{B}_{1}$ is also known as thiamine because the molecule contains partly sulphur, partly amino group. Due to its antineuritic properties, it is also called aneurine. This is the most heat sensitive member of the B-group vitamins. It is quickly inactivated in the presence of heavy metal salts, oxidizing agents, and sulphuric acid. The husk and germ of cereal grains is a particularly rich vitamin source, wherefore bread made from wheat flour contains vitamin $\mathrm{B}_{1}$ in very small quantities as opposed to brown bread. The different tissues (kidney, liver, and muscle tissues) contain vitamin $\mathrm{B}_{1}$ in varying quantities depending on the animal species. Yeast disposes of the highest thiamine content. The average diet barely covers the $1.5-2.0 \mathrm{mg}$ daily thiamine requirement of an adult person.

Although a varied diet can cover the vitamin $\mathrm{B}_{1}$ needs of the elderly, experience shows that a great number of elderly people has a poor supply of this vitamin. In an experiment, $46 \%$ of the participating elderly individuals had vitamin $\mathrm{B}_{1}$ deficiency as they had been consuming one third less of this vitamin than RDA specifications (Abraham et al., 1979). $5 \%$ of retirement home residents and $13-43 \%$ of community home residents are poorly supplied with vitamin $\mathrm{B}_{1}$ (Joshi \& Morley, 2006). The reason for such a low percentage of deficiency in retirement home residents is that individuals at risk are regularly provided with multivitamin supplements, stocking them with sufficient quantities of vitamin $B_{1}$.

While vitamin $B_{1}$ supplements several times the RDA value were shown to improve comprehension, another experiment did not yield any improvement in elderly people with Alzheimer's disease. Generally speaking, vitamin $\mathrm{B}_{1}$ supplements have made a valuable contribution to improving the elderly population's quality of life, a fact demonstrated by improved appetite, restful sleep, higher level of physical activity, and reduced level of fatigue. Quantities several times the RDA value are well tolerated by the organism since the excess is eliminated in urine.

In industrialized countries, vitamin deficiency often has its roots in alcoholism, as with the high amount of fluid intake vitamin $\mathrm{B}_{1}$ is expelled from the system via elimination through the kidney (Suter et al., 2000). Vitamin $\mathrm{B}_{1}$ deficiency can also be traced back to kidney problems for haemodialysis or peritoneal dialysis can accelerate the elimination of vitamin $\mathrm{B}_{1}$ from the body (Hung et al., 2001). 


\subsection{Vitamin $\mathrm{B}_{2}$}

In the human organism, vitamin $\mathrm{B}_{2}$ (riboflavin, lactoflavin) is the cofactor of flavin mononucleotide (FMN) and of flavin adenine dinucleotide (FAD), wherefore it takes part in the biological oxidation processes, the mitochondrial oxidative phosphorylation, and the synthesis of adenosine triphosphate (ATP). These enzymes are collectively termed as flavoproteins. Entering into reaction with the substrate, it reversibly binds two hydrogen atoms on the two nitrogen atoms, during which flavin is reduced to leucoflavin. It plays a vital role in the production of reduced glutathione, an excellent reduction agent of the organism. It also takes part in the detoxification processes in the liver, through which the body neutralizes toxic substances and various drugs.

Vitamin $\mathrm{B}_{2}$ deficiency causes inflammatory symptoms and cracking to appear on the mucous membrane of the mouth and tongue, while general fatigue and visual disturbances also manifest themselves. This deficiency is present in many places all over the world in people who struggle with persistent diarrhoea, have liver problems, or are alcoholics. There are rare cases of specifically vitamin $\mathrm{B}_{2}$ deficiency; much rather, the combined deficiency of several members of the B-group vitamins triggers clinical symptoms.

There are few studies on the elderly people's vitamin $B_{2}$ requirements. Broadly speaking, the oxidative damage of the lens proteins can be prevented by increasing the nutrients' antioxidant components - in this context, the risk of cataract development was shown to be inversely proportional to riboflavin intake (Cumming et al., 2000). It has also been established that in the prevention of cataract development not only riboflavin but all compounds with antioxidant properties introduced into the body are efficient.

Vitamin $\mathrm{B}_{2}$ is not sensitive to heat, but it easily decomposes upon exposure to light, via a photochemical reaction entailing the separation of the side chain and resulting in the production of the biologically inactive alloxasine derivatives. Riboflavin is widely spread in plant and animal tissues as well as in various foodstuffs. Vitamin $\mathrm{B}_{2}$ content is especially high in liver, kidney, fish, egg, milk, and different vegetables. Milk is particularly rich in this vitamin, which is why it is also called lactoflavin. Humans' daily vitamin $\mathrm{B}_{2}$ requirements are $1.5-2.0 \mathrm{mg}$.

\subsection{Vitamin $\mathrm{B}_{3}$}

The deficiency disease of vitamin $\mathrm{B}_{3}$ (nicotinic acid amide, niacin, vitamin PP) is pellagra, which first of all occurs in people who mostly consume corn-based foodstuffs. This disease starts off with symptoms of general fatigue, then a 
dysfunction of the gastrointestinal tract develops, inflammatory cracking appears on the mucous membrane of the mouth and tongue, and the skin surface becomes rough, inflammatory, and exfoliative. The above listed symptoms will subside if nicotinic acid amide is administered, but tryptophan, thiamine, and riboflavin intake are also necessary to achieve a complete recovery.

Regarding its chemical structure, vitamin PP is nicotinic acid, also called niacin, whereas in natural substances we can find nicotinic acid amide, i.e. niacinamide. The living organism can easily amidate nicotinic acid. Niacin is widely spread in nature for it can be found in its coenzyme form in all living cells. High amounts of it can be found in the husk of cereal grains, in yeast, liver, kidney, the meat of animals and fish, milk, egg, and vegetables. Some of the niacin can be found in protein-bound form, and therefore this part of the cereals' niacin content - since a release cannot take place but via alkaline hydrolysis - will not be bioavailable by way of nutrient intake (Gregory, 1998). Corn has very small amounts of nicotinic acid amide and tryptophan, leading to frequent cases of pellagra in those living on a corn-based diet.

Nicotinic acid amide is integrated into the dinucleotide coenzyme (NAD, NADP) of the pyridine enzymes. As one of the double bonds is disrupted, the pyridine ring takes up hydrogen, this way becoming part of the enzymatic action. Besides this, it has a vital role in producing the hydrochloric acid of the gastric juice and lowering cholesterol levels in the blood, while also having vasodilatory effects. In the presence of vitamins $\mathrm{B}_{1}, \mathrm{~B}_{2}$, and $\mathrm{B}_{6}$, the liver is able to synthesise it from tryptophan, but with a very low conversion efficiency as for the biosynthesis of $1 \mathrm{mg}$ of niacin $60 \mathrm{mg}$ of tryptophan is required.

The daily nicotinamide requirement of an adult person is $10-20 \mathrm{mg}$. Its RDA value for vitamin $B_{3}$ is $14 \mathrm{mg} /$ day for women over 50 years of age and $16 \mathrm{mg} /$ day for men of the same age-group. Gastric ulcer, enteritis, or alcoholism may significantly increase these values. For treating cardiovascular symptoms and, formerly, reducing the cholesterol level in the serum, doses of 500-3,000 mg/day were efficiently applied. It has now become clear that an increased quantity of vitamin $\mathrm{B}_{3}$ increases the high-density lipoprotein level (HDL) in the serum, reduces triglyceride and lipoprotein(a) levels, and - to a lesser degree - reduces the level of low-density lipoprotein (LDL) as well as the total amount of cholesterol (Brown et al., 2001). When administered alone, vitamin $\mathrm{B}_{3}$ reduced the rate of cardiovascular diseases (Canner et al., 1986). Medication aimed at reducing LDL cholesterol level is more efficient if administered along vitamin $\mathrm{B}_{3}$. 


\subsection{Vitamin $\mathrm{B}_{6}$}

Hypervitaminosis of vitamin $\mathrm{B}_{6}$ (pyridoxine) causes disturbances in protein metabolism. Its deficiency induces symptoms reminding of pellagra: the mouth and the eyes turn red, inflammation appears, the skin becomes chapped and exfoliative, and hair falls out. The term pyridoxine comprises three compounds with similar vitamin effects: pyridoxol, pyridoxal, and pyridoxamineall of them being substituted pyridine derivatives. Pyridoxol can be found in foods of plant origin, whereas pyridoxal and pyridoxamine in foods of animal origin as phosphate ester. Phosphoric acid is linked to the primary alcoholic hydroxyl group at position 5 , and so vitamin $\mathrm{B}_{6}$ exerts its physiological effect in the form of pyridoxal-5-phosphate (PLP).

It plays a biological role in the intermediary metabolism of the amino acids, where its phosphoric acid esters are the coenzymes of various enzymes (aminotransferases, amino acid decarboxylases, desulphurylases, etc.). These enzymes take part in the synthesis of amino acids and neurotransmitters (serotonin, norepinephrine, $\gamma$-amino butyric acid). PLP is required for the synthesis of nicotinic acid from tryptophan and it is the coenzyme of glycogen phosphorylase, which releases glucose from glycogen; it takes part in glycogenesis as well, during which sugar is created from amino acids.

Meat, fish, liver, egg yolk, whole grains, vitamin-supplemented cereals, leafy vegetables, leguminous plants are all sources of vitamin $\mathrm{B}_{6}$, but intestinal bacteria also produce it. Daily requirements of an adult person amount to 2-3 mg, but as we get older these needs may be higher. Vitamin $\mathrm{B}_{6}$ of plant origin can be found in glycosidic linkage, whose availability is limited. It is absorbed in the first segment of the small intestine via passive transport, which is aided by the acidic environment. Since our body cannot store it, a regular intake is necessary on a daily basis. Vitamin $\mathrm{B}_{6}$ deficiency occurs when consuming vitamin-deficient foods, in cases of cirrhosis, inadequate dialysis, or with alcoholics. Certain medications can also trigger vitamin deficiency, in which case dietary intake must be supplemented with vitamin $B_{6}$.

Along with other digestive problems, vitamin $\mathrm{B}_{6}$ deficiency often occurs in elderly people (Johnson, 1995), increasing the homocysteine level in the serum, which is generally acknowledged to increase the risk of cardiovascular diseases (Eikelboom et al., 1999). Several researchers reported vitamin $\mathrm{B}_{6}$ intake in quantities much larger than the RDA value to protect against cardiovascular diseases and high cholesterol level, while also having beneficial effects for diabetic patients. The co-administration of vitamin $\mathrm{B}_{6}$ and folic acid has probably reduced the incidence of coronary artery disease (Folsom et al., 1998). 
In the case of vitamin $\mathrm{B}_{6}$ deficiency, the immune system can also be compromised as the affected person may have to face a considerably low lymphocyte count and likewise a low interleukin production. It has been established that the normal functioning of the immune system can be restored by applying several times the recommended RDA value (Meydani et al., 1991). A close negative correlation was found in the elderly between cognitive abilities, high homocysteine as well as low vitamin $\mathrm{B}_{6}$ levels. With doses of vitamin $\mathrm{B}_{6}(20$ $\mathrm{mg} /$ day) exceeding RDA values $(1.7 \mathrm{mg} /$ day), a definite improvement was observed regarding the memory-related abilities of elderly people aged 70-79 years (Bryan et al., 2002).

The excess of vitamin $\mathrm{B}_{6}$ is oxidized into pyridoxine acid, and is eliminated from the body.

\subsection{Pantothenic acid}

Pantothenic acid deficiency causes fatigue, agitation, muscle cramps, and indigestion. Its avitaminosis inhibits growth, reproduction, causes pellagra-like mutations, subcutaneous bleeding, and neural dysfunctions. Pantothenic acid is in fact the peptide of the pantoic acid, formed by beta-alanine. It is an acidic water-soluble compound, which under neutral conditions is resistant to light and the oxygen in the air. It is inactivated upon exposure to strong acidic or alkaline substances. Natural occurrences are also observed in the case of pantothenol, which is the alcohol analogue of pantothenic acid, and pantotheine, which is the decarboxylation product of pantothenol-cysteine resulting from the linkage of pantothenic acid and cysteine. Both of the compounds act as pantothenic acid.

Pantothenic acid performs its biological effect in metabolism as a constituent part of coenzyme A, acting as an indispensable contributor to the utilization of energy-providing nutrients and also controlling the interconversion of fats and carbohydrates. Coenzyme $\mathrm{A}$ is in fact created through the union of pantetheine and ADP. We have knowledge of more than 70 enzymatic reactions in which it takes an active part. The best known of them are the transfer of the acyl group during the decomposition of carbohydrates, fatty acids, and amino acids and the participation in the biosynthesis of fatty acids as well as porphyrin and steroid compounds.

\subsection{Folic acid}

Folic acid (folate, pteroylglutamic acid) occurs in high quantities in the leaves of green plants. Its deficiency in the human organism causes anaemia since 
folic acid along with vitamin $\mathrm{B}_{12}$ regulates the formation of red and white blood cells and of platelets. Additionally, it plays a role in developing the mucous membrane of the gastrointestinal tract. The term folic acid is used for a class of compounds whose parent compound is pteroic acid and the 1, 3 , or 7 glutamic acid molecules linked to it. Pteroic acid is the derivative of para-aminobenzoic acid and pteridine. It is the first glutamic acid linked through amide bond, while the rest are linked through $\gamma$-peptide bond - they are termed pteroylglutamic acid, pteroyltriglutamic acid, and pteroylheptaglutamic acid. Induced by ascorbic acid and NADPH, pteroylglutamic acid forms 5,6,7,8-tetrahydrofolic acid, which will biologically transform into active folinic acid.

Folinic acid takes part in building up the coenzyme of synthetases, transferases, and isomerases. Enzymes play an active role in the transfer of onecarbon units; as carbon donors, they are essential in the synthesis of amino acids and nucleic acids as well. One of the most important processes is homocysteine remethylation to methionine, which requires folic acid and vitamin $\mathrm{B}_{12}$. In their absence, the homocysteine level in the serum increases, just as the risk of developing cardiovascular diseases.

Folic acid can be absorbed from the small intestine both via passive and active transport. From the liver, it re-enters the bloodstream, while the excess is eliminated from the body via the kidney, wherefore our organism cannot store larger quantities of folic acid. Following the reduced intake of folic acid, its deficiency will manifest within a few days although clinical symptoms take a lot more time to appear. The incidence rate of folic acid deficiency in the elderly population is $2-34 \%$ (Joshi \& Morley, 2006). Besides a vitamin-deficient diet and malabsorption, excessive alcohol consumption, smoking, atrophic gastritis, enteritis, and certain medications can also contribute to a state of avitaminosis (Kishi et al., 1997). Since folic acid deficiency in the elderly can be linked to the development of diseases such as various types of cancer, arteriosclerosis, or senile dementia, the relevant daily RDA value was increased to $400 \mu \mathrm{g}$. A daily intake of $5 \mathrm{mg}$ folic acid was shown to reduce the development of colon cancer and other intestinal tumours (Kim et al., 2001; Su \& Arab, 2001). Two servings of alcohol per day coupled with folic acid deficiency also increased the risk of developing colon cancer. An increased folic acid supplement reduced the incidence of developing breast cancer in alcoholic women (Rohan et al., 2000).

Recently, there have been several studies on the connection between folic acid intake and the deterioration of cognitive abilities in elderly people. Researchers pointed out that folic acid deficiency in people aged over 65 years 
is accompanied by short-term memory impairment, which is a form of senile dementia (Ebly et al., 1998). Upon administering folic acid supplement, a reduced risk of developing ischemic stroke can be observed (He et al., 2004).

People's normal diet usually contains sufficient amounts of folic acid. The food sources richest in folic acid are liver, kidney, meat, various mushrooms, asparagus, Brussels sprouts, citrus fruits, and leafy vegetables. Folates are highly sensitive to oxidation and heat; therefore, in the process of preparing food, 50-95\% of the basic material decomposes, but folic acid - the biologically active form of the vitamin - is essentially more stable (Johnson et al., 2002). Intestinal flora also contributes to the $0.4 \mathrm{mg}$ of folic acid necessary for the normal functioning of the human organism.

There are no known adverse effects of excessive folic acid intake, but its consumption in high quantities over a longer period of time can lead to vitamin $\mathrm{B}_{12}$ deficiency and may even cause irreversible neurological damage, which is why a daily folic acid intake exceeding $1,000 \mu \mathrm{g}$ is not advised (Higdon, 2002).

\subsection{Biotin}

The avitaminosis of biotin (vitamin $\mathrm{H}$ ) causes loss of appetite, dermatitis, hair loss, and greasy skin surface. It is an important growth factor for saccharomyces cerevisiae as well. The biotin molecule is made up of a sulphurous annular portion and valeric acid side chain. The structure made up of two 5 -membered heterocycles is formed through the linkage of carbamide and thiophene ring. Biotinal, the aldehyde version is also biologically active and can be oxidized to biotin. We can also find in nature its acid amide formed with lysine, the likewise active biocytin.

Biotin is not heat sensitive, but it decomposes upon exposure to strong acidic and alkaline substances or oxidizing agents, while it is slowly inactivated when exposed to light. Avidin glycoprotein found in raw egg white forms such a complex with biotin that can resist enzymatic breakdown, and thus the avidin-biotin complex is eliminated from the body through the digestive tract. Biotin can be found in plants in free form, while in animal tissues, microorganisms, and dairy products its protein-bound form can be observed. Humans' daily requirement amounts to $100-300 \mu \mathrm{g}$. In terms of human nutrition, the most important sources are represented by liver, kidney, milk, egg yolk, soy, vegetables, nut, and yeast, while intestinal flora can also synthesise biotin. Specialized literature does not mention any data on elderly people's biotin needs. 
Its biological function is carried out in the role of the enzymatic prosthetic group, linked to the lysine part in the enzyme protein peptide chain. Biotincontaining enzymes take part, first of all, in the decarboxylation, deamination, carboxylation, and synthetisation processes essential in carbohydrate and lipid metabolism.

\subsection{Vitamin $\mathrm{B}_{12}$}

Human organism needs vitamin $\mathrm{B}_{12}$ (cobalamin) for a normal growth, a healthy nervous system, and haematopoiesis. Its central structure is the corrin ring, similar to the porphyrin shell and forming a girdle around the cobalt atom. Via complex bonding, 5,6-dimethylbenzimidazole and a cyanide or a hydroxyl or a nitrite radical are linked to the cobalt atom. All three forms have vitamin effects, since following absorption these radicals are equally replaced by 5 -deoxyadenosine, and cobalamin is incorporated into the enzyme in this form (adenosylcobalamin) (Higdon, 2003).

It takes part as a coenzyme in several important processes (thiamine synthesis, reduction of one-carbon units, propionic acid metabolism, etc.). In several biochemical processes, it is present together with folic acid. It is the cofactor of methionine synthase and L-methylmalonyl-CoA mutase. The former enzyme converts homocysteine into methionine, which is required for the synthesis of S-adenosylmethionine, and it takes part as a methyl group donor in a number of biochemical reactions where the presence of a methyl group is necessary, such as RNA and DNA methylation. It controls the synthesis of L-methylmalonyl-CoA mutase and succinyl-CoA from methylmalonyl-CoA.

A lesser degree of vitamin $B_{12}$ deficiency causes neurological disorders, whereas high levels of vitamin $\mathrm{B}_{12}$ deficiency lead to pernicious anaemia, whose typical symptoms are reduced red blood cell count and the unnatural swelling these cells; additionally, loss of appetite, weakness, and indigestion can also occur. The absorption of vitamin $\mathrm{B}_{12}$ requires mucoprotein produced in the gastric wall and with a molecular weight of 60,000 daltons, which can release vitamin $\mathrm{B}_{12}$, introduced into the body through dietary intake, from protein complexes, and helps the linking of appropriate receptors in the small intestine.

Vitamin $\mathrm{B}_{12}$ is exclusively manufactured by microorganisms - it cannot be found in plants and yeast, while the intestinal microorganisms satisfy the needs of herbivorous animals. The consumption of foods of animal origin with high protein content helps humans to the necessary vitamin $\mathrm{B}_{12}$, regarding which the daily requirement is $3-4 \mu \mathrm{g}$. In the case of elderly people, the RDA value for vitamin $\mathrm{B}_{12}$ is $2-4 \mu \mathrm{g}$, about half of which can be covered by consuming 
half a litre of milk or a corresponding dairy product. Milk is an extremely important source of vitamin $\mathrm{B}_{12}$ for vegetarians too as without it they cannot obtain this essential substance.

Gastric acid and enzymes are required in order for vitamin $\mathrm{B}_{12}$ to be released from its bonds and become available via absorption. Pancreatic proteases break down proteins, and as a consequence the released vitamin $\mathrm{B}_{12}$ enters the bloodstream, and through a transport mechanism gets into the liver, where it is stored up. Vitamin $\mathrm{B}_{12}$ deficiency in the elderly - except for vegetarians - can be associated with low levels of vitamin $\left(\mathrm{B}_{12}\right)$ absorption. Since the human body is able to store it in the liver, where a sort of a rescue reaction is also present, it has a long half-life in this organ, and therefore deficiency diseases do not develop until a few years' time. It is estimated that $4-43 \%$ of the elderly population suffers from vitamin deficiency.

As gastric acid and digestive enzymes are needed to release vitamin $\mathrm{B}_{12}$ from protein, gastric acid deficiency leads to vitamin deficiency. Gastritis is very frequent in elderly people $(30 \%)$ : it reduces absorption, which leads to vitamin deficiency. High bacteria count in the small intestine due to gastric acid deficiency can also lead to vitamin deficiency, as bacteria use up vitamin $\mathrm{B}_{12}$ for reproduction. Vitamin $\mathrm{B}_{12}$ deficiency in the elderly population can occur consequent upon pancreatic enzyme production activities or following gastrointestinal surgery (Joshi \& Morley, 2006). This may cause functional disturbances of the central nervous system, forgetfulness, and depression in the elderly.

As an established procedure, cyanocobalamin intramuscular injection is prescribed for cases of vitamin malabsorption. As the body is capable of storing it, a dose of $1,000 \mu \mathrm{g}$ administered every month or every few months may be the solution to the problem (Hajjar et al., 2000), though not a preferred procedure anymore. For explicit anaemia, it is recommended that the patient take $1,000 \mu \mathrm{g}$ of vitamin $\mathrm{B}_{12}$ instead of the usual daily doses of 100-500 $\mu \mathrm{g}$. Similar to vitamin $\mathrm{B}_{6}$ deficiency, low levels of vitamin $\mathrm{B}_{12}$ also increase the homocysteine level in the serum, enhancing susceptibility to cardiovascular diseases. Like with folic acid, $500 \mu \mathrm{g} /$ day of vitamin $\mathrm{B}_{12}$ supplement could also reduce the homocysteine level significantly (Stabler et al., 1997).

Decreasing levels of vitamin $\mathrm{B}_{12}$ in the serum were accompanied by increasing homocysteine levels, which could be successfully compensated for by an amount of vitamin $\mathrm{B}_{12} 3-5$ times the $\mathrm{RDA}$ value. Vitamin $\mathrm{B}_{12}$ deficiency was associated with senile Alzheimer's disease and showed a strong correlation with depression symptoms over 65 years of age. 


\section{$3.9 \quad$ Vitamin $\mathrm{B}_{15}$}

Vitamin $\mathrm{B}_{15}$ (pangamic acid) is an essential methylating agent of the living organism. Its physiological importance is due to the role it plays in assisting tissue oxygen metabolism, while also having detoxifying and lipotropic effects. Those substances have lipotropic properties that prevent certain organs (the liver) from lipomatosis. Its chemical composition: it is an ester of D-gluconic acid and dimethylglycine. In our foodstuffs, it can be found in large quantities in liver, yeast, and treacle. There are no data recorded in literature on elderly people's pangamic acid requirements.

\subsection{Vitamin U}

Vitamin U (S-methylmethionine) cures gastric ulcer as well as inhibits its formation, lowers lipid and cholesterol levels in the serum, and its lipotropic effect is similar to that of pangamic acid. In terms of chemical composition, it is the sulphur-methylated, L-configuration, basic, reactive sulphonium derivative of methionine. In higher plants, it is formed from S-adenosylmethionine. In the human organism, it takes part in the synthesis of choline and creatine. It is an essential methylating agent, a substitute for methionine. The human body cannot synthesise a methyl group, which is why it needs methyl donors to break down fats. Vitamin $U$ can be found in cabbage, lettuce, tomato, spring onion, radish, leaf parsley, asparagus, and fruits. As a result of cooking, it breaks down into dimethyl sulphide and homoserine; therefore, raw vegetables and their expressed juices are in particular rich sources of vitamin U. There are no data recorded in literature on elderly people's vitamin U requirements.

\subsection{Vitamin $\mathrm{C}$}

Scurvy developed as a consequence of vitamin C (ascorbic acid) deficiency was a disease feared by everyone in the Middle Ages, but survey results showed that scurvy is actually the outcome of the combined deficiency of vitamin $\mathrm{C}$ and certain bioflavonoids. Its characteristic signs are general fatigue, shortness of breath, cardiac dysfunction, pain in the muscles and bones, increased gingival bleeding, and then petechial haemorrhaging on the lower extremities caused by the vulnerability of the capillary vessels and increased capillary permeability. The bones become fragile, the joints swell up, the teeth become loose and fall out, wounds heal very slowly, and finally death sets in. Nowadays, vitamin $\mathrm{C}$ deficiency would only cause the development of the so-called spring fever, consequent upon which the immunity of the body decreases and at the same time susceptibility to colds increases. 
Scurvy can develop only as an outcome of vitamin C intake not exceeding $10 \mathrm{mg} /$ day throughout a period of at least three months. This disease usually goes along with poor-quality dentine, which will cause the teeth to fall out. In the case of vitamin $\mathrm{C}$ deficiency, iron-deficiency anaemia can also occur.

The abovementioned symptoms and diseases come as a corollary to the fact that vitamin $\mathrm{C}$ is necessary for the synthesis and integrity of the collagen, a crucially essential contributor to the development of blood vessels, tendons, and ligaments as well as to wound healing. Vitamin $\mathrm{C}$ deficiency results in abnormal collagen cross-linking, reducing its tensile strength (Joshi \& Morley, 2006). Altered collagen structure may lead to abnormal bone formation. It also plays an important role in norepinephrine and carnitine synthesis. Carnitine helps fatty acids enter the mitochondria, this way assisting energy release. Vitamin $\mathrm{C}$ is highly efficient in fighting off free radicals attacking the body, which are formed in our everyday life as a consequence of contaminants, toxic substances or as a result of smoking. Vitamin C protects our DNA, proteins, unsaturated fatty acids, and carbohydrates from oxidation. It helps the duodenal absorption of iron and other microelements, the reduction of methemoglobin to haemoglobin, and it reduces the nitrates, this way inhibiting the development of mutagenic nitrosamines, and thus preventing cancer.

The average daily vitamin $\mathrm{C}$ requirement for an adult person, depending on the (type and amount of) work carried out, is cc. $80-100 \mathrm{mg}$. Although the excess of vitamin $\mathrm{C}$ is eliminated in urine, its excessive consumption is detrimental to health (due to the formation of renal calculi). The experiments of Svirberly and Szent-Györgyi (1932) demonstrated that administering ascorbic acid on its own would not put an end to the increased permeability of blood vessels, haemophilia. In order for this to happen, we need the help of vitamin $\mathrm{P}$, first manufactured in 1936 for the regulation of permeability. Bioflavonoid glycosides have vitamin $\mathrm{P}$ effects, and among them rutin proved to be biologically the most active.

Rutin is a glycoside whose sugar moiety is rutinose and its aglycone is a three-ringed flavonol compound. Rutinose is an oligosaccharide formed from D-glucose and L-rhamnose via a $\beta$-configuration binding. (Bioflavonoids are not included in the category of vitamins anymore.)

Given that it is a water-soluble vitamin, excessive vitamin $\mathrm{C}$ intake can have very few adverse effects. However, a daily vitamin $\mathrm{C}$ intake of over 2,000 mg can lead to gastrointestinal symptoms such as nausea, abdominal cramps, and diarrhoea. Abnormal vitamin $\mathrm{B}_{12}$ absorption and elevated blood oestrogen levels may also occur in these cases (Miller \& Hays, 1982). It is considered by many a medicine against common cold although experiments have shown 
that its consumption in daily doses of $2,000 \mathrm{mg}$ has no appreciable effect on mild cases of cold, but it has reduced the duration of common cold diseases in adult individuals by $8 \%$.

Some claim that high doses of vitamin $\mathrm{C}$ intake help prevent the onset of cardiovascular symptoms as well as cancer in older people, but these allegations have not yet been confirmed. However, it appears to be certain that it works as an efficient factor in preventing the opalescence of the lens among members of the senior population (Mares-Perlman et al., 2000). Since the beneficial effects of vitamin $\mathrm{C}$ megadosage are not evident, the administration of such large doses is not practical. Based on current knowledge, a daily vitamin $\mathrm{C}$ intake of 100-140 mg seems to be a sufficient amount for this vitamin to access all essential points in our body and prevent any harmful processes caused by its deficiency. By consuming foods of natural origin rich in vitamin $\mathrm{C}$ (five servings of fruits and vegetables per day), we may even achieve a daily vitamin C intake of $200 \mathrm{mg}$, which can significantly reduce the risk of cancer in elderly people.

Despite there is no biochemical system in our body that could store vitamin $\mathrm{C}$, adrenal glands, the pituitary gland, the thymus, and the retina may contain as much as 50 times the amount found in the serum. The brain, the placenta, the testicles, the thyroid gland, the pancreas, and the kidneys all contain 5-25 times more vitamin $\mathrm{C}$ than the serum (Jakob, 1999). Generally speaking, it can be concluded that women's serum contains $20 \%$ more vitamin $\mathrm{C}$ compared to men and that non-smokers are better supplied in this regard than their smoking peers. Consequently, the RDA value for men is $90 \mathrm{mg} /$ day, for women $75 \mathrm{mg} /$ day, while for smokers is $35 \mathrm{mg}$ /day more than for non-smokers (Johnson et al., 2002).

Vitamin $\mathrm{C}$ is the L-configuration lactone of the 2-keto-gulonic acid, the oxidation product of glucose. The 2-keto-gulonic acid also has vitamin effects, but the biological effect of D-ascorbic acid is insignificant. Dienyl group is typically found with ascorbic acid and it can be oxidized to diketo group, wherefore ascorbic acid is a strong reducing agent; as a characteristic feature, it is reversibly oxidized to dehydroascorbic acid together with which it forms a redox system. Both forms have vitamin effects, but ascorbic acid is considered to be the more valuable product. Ascorbic acid is converted into diketogulonic acid via oxidation, which, in its turn, breaks down into oxalic acid and Lthreonic acid, and these are irreversible transformations. Ascorbic acid is oxidized not only upon exposure to the oxygen in the air or certain chemicals but also by certain enzymatic reactions. Vitamin inactivation is catalysed by heating, light, and trace metals. In the presence of amino acids, ascorbic 
acid, dehydroascorbic acid, and its various decomposition products may be converted via Maillard reactions into brown-coloured products. Ascorbic acid typically has a sour taste, it is a crystalline material easily soluble in water, the $\mathrm{pH}$ value of its $2 \%$ aqueous solution is 2.8 , its acidic solutions are stable, but in the presence of air it decomposes above $\mathrm{pH} 4$. The hydrogen of its hydroxyl group on the second carbon atom $\left(\mathrm{pK}_{1}=4.2\right)$ dissociates easily, while the one on the third carbon atom dissociates hardly $\left(\mathrm{pK}_{2}=11.6\right)$. Its primary alcoholic hydroxyl group can be esterified with fatty acid; its ester formed with palmitic acid, ascorbyl palmitate, has antioxidant effect in fats. The stereoisomers of ascorbic acid (D-ascorbic acid, D-isoascorbic acid, Daraboascorbic acid) are antioxidants without any vitamin effects.

The artificial chemical synthesis of vitamin $\mathrm{C}$ starts out from D-glucose, followed by the conversion of D-sorbitol into L-ascorbic acid. Green pepper, tomato, cabbage, blackcurrant, fresh and dried rose hip, potato, parsley, and the various tropical fruits particularly abound in vitamin C. Among animal tissues, only offal contains considerable quantities of vitamin $\mathrm{C}$, while the vitamin $\mathrm{C}$ content of cow milk is again very low. The vitamin $\mathrm{C}$ content of foodstuffs depends on the type of food, the season, the food production technology, and especially the type of heat treatment; therefore, heat-processed vegetables and vegetable soups are not exactly proper sources of vitamin C (Johnson et al., 2002). During storage, the vitamin C content of leafy vegetables can drop by 50-80\% compared to their fresh counterparts.

Vitamin $\mathrm{C}$ is absorbed from the small intestine via energy-intensive active transport. Its absorption and bioavailability are inversely proportional to the amount of intake. When administered in small doses, vitamin $\mathrm{C}$ has a higher bioavailability, while absorption is also influenced by the composition of the food consumed. If we increase the dosage, absorption will decrease although the amount absorbed will increase due to the larger doses (Levine et al., 1999). The half-life of vitamin $\mathrm{C}$ in the human organism is very short: 30-60 minutes. The low vitamin content of the serum is derived either from reduced absorption or rapid elimination. Absorption may be affected by pharmaceuticals such as aspirin, which was shown to reduce the vitamin $\mathrm{C}$ content of white blood cells by $50 \%$, a fact that can be accounted for by the elimination via the kidney.

The biological effect of ascorbic acid is closely related to its oxidationreduction potential. In the digestive tract, it stimulates iron and calcium absorption, and it takes part in cellular biochemical processes by maintaining the reduced condition and acting as hydrogen donor. It contributes to connective tissue collagen formation, the synthesis of adrenal hormones, serotonin tissue hormone production, and the oxidative degradation of tyrosine. 


\section{Conclusions}

Our body cannot store up water-soluble vitamins, with the sole exception of vitamin $\mathrm{B}_{12}$, and therefore various doses of vitamin intake are necessary on a daily basis or, in some cases, several times a week. Water-soluble vitamin intake is also important for the elderly population because many of them are highly efficient against free radicals and due to their antioxidant properties they slow down the ageing process. Some specialists urge the elderly to consume vitamins many times the daily requirements, which is, however, reasonable only during certain diseases; otherwise, the suggested quantities would verge upon hypervitaminosis.

Vitamin B deficiency rarely occurs among the elderly in developed countries as, for the most part, foods can cover vitamin needs and elderly people take vitamin tablets on a daily basis, which contain these in optimal quantities. Vitamin deficiency can be caused by alcoholism, on the one hand, since the high volume of fluid leaving the body eliminates large amounts of vitamin via the kidney, and by different kidney problems, on the other hand, these being related to the pathological nature of dialysis.

Almost all members of the B-group vitamins were established to have beneficial effects on the body in cases of slight overdose or, occasionally, even multiple overdose. Vitamin $B_{1}$ supplement was shown to improve cognitive abilities in elderly people, increase their activity level and appetite, relax their sleeping, and altogether contribute to improving the quality of life. The human organism proved to have a good toleration for amounts several times the RDA specifications. Vitamin $B_{2}$ supplement can help prevent the oxidative damage of lens proteins and the development of cataract.

Niacin requirements can significantly increase in old age in cases of gastric ulcer, enteritis, or alcoholism. Supplement intake of more than ten times the necessary amount will increase the level of high-density lipoprotein in the serum, whereas it will decrease the concentrations of low-density lipoprotein, triglyceride, and total cholesterol. When administered alone, it reduced the incidence of cardiovascular diseases and the efficiency of medications lowering LDL cholesterol level.

Vitamin $\mathrm{B}_{6}$ deficiency may exhibit a higher frequency with elderly people and, accompanied by indigestion, increases the homocysteine level in the serum, which entails an increased cardiovascular risk. Its consumption in quantities well above the daily requirement lowers the cholesterol level in the serum, has positive effects in diabetics, and protects against cardiovascular diseases. Its overdose can help restore the normal functioning of a compromised 
immune system and has shown a marked improvement in terms of memory in elderly people. If administered together with folic acid, it improves coronary artery condition.

Pantothenic acid deficiency in the elderly can occur rarely, mostly in cases of malnutrition. The primary causes of folic acid deficiency are malnutrition, malabsorption, alcoholism, atrophic gastritis, enteritis, and certain medications. Elderly people's daily needs can be covered with doses of $0.4 \mathrm{mg}$, but increasing this amount to $5 \mathrm{mg}$ can reduce the risk of colon cancer, other malignant tumours, arteriosclerosis, and senile dementia. Folic acid supplement intake reduces ischemic stroke and the risk of short-term memory impairment. Its excessive consumption is not recommended since that would lead to vitamin $B_{12}$ deficiency and irreversible neurological damage.

Biotin deficiency does not usually occur in old age as foods contain it in sufficient amounts and intestinal flora synthesises it too. In the case of a mixed diet containing foods of animal origin in sufficient amounts, vitamin $\mathrm{B}_{12}$ deficiency does not occur as a rule. What causes deficiency in elderly people is that normally gastric acid and enzymes help the vitamin to release from its bindings prior to absorption, which mechanism, however, does not work properly in old age. Entering the liver, it is stored up; consequently, vitamin deficiency is only to be reckoned with if the gastrointestinal absorption of the vitamin decreases. With vitamin storage level at its maximum, deficiency diseases may not even develop but in several years' time. In cases of vitamin $\mathrm{B}_{12}$ deficiency, functional disturbances of the central nervous system, depression, and forgetfulness may occur, while due to the increased serum homocysteine level the risk of developing cardiovascular diseases may also become higher. Vitamin $\mathrm{B}_{12}$ and $\mathrm{U}$ deficiency occurs in extremely rare cases involving elderly people.

As for vitamin $\mathrm{C}$ efficiency, the beneficial or adverse effects of its overdose, the results are highly contradictory. Some claim that high doses $(1,000 \mathrm{mg}$ or more per day) of vitamin $\mathrm{C}$ intake help prevent the onset of cardiovascular symptoms and cancer in elderly people, whereas others have not found such beneficial effects. It appears to be certain that several times the required dosage is efficient in preventing the opalescence of the lens, but a daily vitamin $\mathrm{C}$ intake exceeding 100-140 mg is not recommended by most. Consumption above $2,000 \mathrm{mg} /$ day may even trigger gastrointestinal symptoms. 


\section{Acknowledgements}

The authors express their grateful thanks for the financial support of the University of Debrecen, Faculty of Agricultural and Food Sciences and Environmental Management, Institute of Food Technology, Sapientia Hungarian University of Transylvania, Faculty of Miercurea Ciuc, Department of Food Science, and the Scientific Research Department of Sapientia Foundation.

\section{References}

[1] G. Brown, J. J. Albers, L. D. Fisher, Regression of coronary artery disease as a result of intensive lipid-lowering therapy in men with high level of apolipoprotein B. New England Journal of Medicine, 323. (1990) $1289-1298$.

[2] J. Bryan, E. Calvaresi, D. Hughes, Short-term folate, vitamin B-12 or vitamin B-6 supplementation slightly affects memory performance but not mood in women of various ages. Journal of Nutrition, 132. (2002) $1345-1356$.

[3] P. L. Canner, K. G. Berge, N. K. Wenger, J. Stamler, L. Friedman, R. J. Prineas, Fifteen year mortality in coronary drug project patients: long term benefit with niacin. Journal of the American College of Cardiology, 8. (1986) 1245-1255.

[4] R. G. Cumming, P. Mitchell, W. Smith, Diet and cataract: the Blue Mountains eye study. Ophthalmology, 107. (2000) 450-456.

[5] E. M. Ebly, J. P. Shaefer, N. R. Campbell, D. B. Hogan, Folate status, vascular disease, and cognition in elderly Canadians. Age and Ageing, 27. (1998) 485-491.

[6] J. W. Eikelboom, E. Lonn, J. Genest, G. Hankey, S. Yusuf, Homocyst(e)ine and cardiovascular disease: a critical review of the epidemiologic evidence. Annals of Internal Medicine, 131. (1999) 363-375.

[7] A. R. Folsom, F. J. Nieto, P. G. McGovern, M. Y. Tsai, M. R. Malinow, J. H. Eckfeldt, D. L. Hess, C. E. Davis, Prospective study of coronary heart disease incidence in relation to fasting total homocysteine, related genetic polymorphisms, and B vitamins: The atherosclerosis risk in communities (ARIC) study. Circulation, 98. (1998) 204-210. 
[8] J. F. Gregory, Nutritional properties and significance of vitamin glycosides. Annual Review of Nutrition, 18. (1998) 277-296.

[9] R. R. Hajjar, R. Stewart, D. Meitzler, The effect of dosing intervals of intramuscular vitamin B12 on serum levels in B12-deficient elderly after loading. Journal of the American Geriatrics Society, 48. (2000) S23.

[10] K. He, A. Merchant, E. B. Rimm, B. A. Rosner, M. J. Stampfer, W. C. Willett, A. Ascherio, Folate, vitamin B6 and B12 intakes in relation to risk of stroke among men. Journal of the American Stroke Association, 35. (2004) 169-174.

[11] J. Higdon, Folic acid. Linus Pauling Institute - Micronutrient Information Center, 2002. http://lpi.oregonstate.edu/infocenter/vitamins/ fa//. Accessed August 8. 2006.

[12] J. Higdon, Vitamin B12. Linus Pauling Institute - Micronutrient Information Center, 2002. http://lpi.oregonstate.edu/infocenter/vitamins/ vitaminB12/. Accessed August 20. 2006.

[13] R. A. Jakob, Vitamin C. In: M. Shils, J. Olson, M. Shike, A. C. Ross (eds), Modern nutrition in health and disease, $9^{\text {th }}$ ed., Williams \& Wilkins, Baltimore, (1999) 467-482.

[14] L. E. Johnson, Vitamin nutrition in elderly. In: J. E. Morley, Z. Glick, L. Z. Rubenstein (eds), Geriatric nutrition: A comprehensive review, $2^{\text {nd }}$ ed., Raven Press, New York, (1995) 79-105.

[15] J. Joshi, J. M. Morley, Vitamins and minerals in the elderly. In: M. S. J. Pathy, A. J. Sincler, J. E. Morley (eds), Principles and practice of geriatric medicine, $4^{\text {th }}$ ed., John Wiley and Sons, Chichester, England, (2006) 329-246.

[16] Y. I. Kim, H. W. Baik, K. Fawaz, Effects of folate supplementation on two provisional molecular markers of colon cancer: a prospective, randomized trial. Journal of Gastroenterology, 96. (2001) 184-195.

[17] T. Kishi, N. Fujita, T. Eguchi, Mechanism for reduction of serum folate by antiepileptic drugs during prolonged therapy. Journal of Neuroscience, 145. (1997) 109-112. 
[18] S. Lawson, J. V. Higdon, B. Frei, The optimum intake of vitamin C: history and controversy. In: H. Asard, J. M. May, N. Smirnoff (eds), Vitamin C. Function and biochemistry in animals and plants. Garland Science/BIOS Scientific Publishers. (2004) 1-6.

[19] M. Levine, S. C. Rumsey, R. Daruwala, Criteria and recommendations for vitamin C intake. Journal of the American Medical Association, 281. (1999) 1415-1423.

[20] J. A. Mares-Perlman, B. J. Lyle, R. Klein, Vitamin supplement use and incident cataracts in a population-based study. Archives of Ophthalmology, 118. (2000) 1556-1563.

[21] S. M. Meydani, J. D. Ribaya-Mercado, R. M. Russel, N. Sahyoun, F. D. Morrow, S. N. Gershoff, Vitamin B-6 deficiency impairs interleukin 2 production and lymphocyte proliferation in elderly adults. American Journal of Clinical Nutrition, 53. (1991) 1275-1280.

[22] D. R. Miller, K. C. Hays, Vitamin excess and toxicity. In: J. N. Hathcock (ed.), Nutritional Toxicology, 1. Academic Press, New York, (1982) 81-133.

[23] L. Pauling, Third case report on lysine-ascorbate amelioration of angina pectoris. Journal of Orthomolecular Medicine, 8. (1993) 137138.

[24] T. E. Rohan, M. G. Jain, G. R. Howe, A. B. Miller, Dietary folate consumption and breast cancer risk. Journal of the National Cancer Institute, 92. (2000) 266-269.

[25] S. P. Stabler, J. Lindenbaum, R. H. Allen, Vitamin B-12 deficiency in elderly: current dilemmas. American Journal of Clinical Nutrition, 66. (1997) 741-749.

[26] L. J. Su, L. Arab, Nutritional status of folate and colon cancer risk: evidence from NHANES I epidemiologic follow-up study. Annals of Epidemiology, 11. (2001) 6-72.

[27] P. M. Suter, J. Haller, J. Hany, W. Vetter, Diuretic use: a risk for subclinical thiamine deficiency in elderly patients. Journal of Nutrition Health and Aging, 4. (2000) 6-71.

[28] J. L. Svirbely, A. Szent-Györgyi, Hexuronic acid as the antiscorbutic factor. Nature, 129. (1932) 576. 